\title{
Characteristic Feature Found in Typical TATARA Product, Japanese Sword
}

\author{
A. K. Das ${ }^{1}$, T. Ohba ${ }^{\text {a1 }}$, S. Morito ${ }^{1}$, G. Takami ${ }^{1}$, T. Fujikawa ${ }^{1}$, M. Yaso ${ }^{2}$ \\ ${ }^{1}$ Department of Materials Science, Shimane University, Nishikawatsu, Matsue, 690-8504, JAPAN \\ ${ }^{2}$ Wakoh Museum, Yasugi-cho, Yasugi 692-0011, JAPAN
}

\begin{abstract}
Tatara is traditional iron making method developed around San-in area in Japan. Typical product of Tatara is Japanese sword, which is tough and strong and is also known as an art object. It is said that Japanese sword cannot be produced without Tatara iron. Thus microscopic observations of Japanese sword attracted researchers and were performed for example by Tawara with optical microscopy at the beginning of 1900s. Since then a few microscopic observations have been performed but not so many. A lot of knowledge of steels has been accumulated and new equipments have been also developed, which give information on atomistic scale. Thus the precise microstructural observations of Japanese sword using such as SEM/EBSD have been made and are compared with ordinary steel. The preliminary experiments indicated that the average martensite block thickness is little longer than that of ordinary steels and crystal orientation distribution is different from ordinary one.
\end{abstract}

\section{Introduction}

A great deal of interest has been found in the recent years to study the micro-structural characteristics of traditional wootz steel specially used in the fabrication of swords. In different parts of the world different types of swords were used as weapons in the medieval age [1]. The extraordinary mechanical properties, exceptionally sharp cutting edge and wavy banding pattern known as damask of those swords are of special interest to the researchers. It is believed that Damascus blades were forged directly from small cakes of steel (named 'wootz') produced in ancient India. A sophisticated thermomechanical treatment of forging and annealing was applied to these cakes to refine the steel to its exceptional quality [2]. On the basis of archeological research and documentation at least three methods of production of ancient steel sword are recognized [3-5]. i) Technique designed for the production of Japanese swords=ii) 'Eastern Damascus' techniques diffused in the Indian-Persian regions and iii) 'Western Damascus' or 'Welded Damascus' which has encountered a significant success in the regions around the Mediterranean Sea. A variety of microstructures and carbon concentration 0.7-2.24 mass\% in different Damascus steel have also been reported [1-5].

The Japanese swords are said to be strong, tough and sharp. The swords in Japan are made from special type of steel called Tamahagane. Tamahagane is produced from iron sand in charcoal bath using a smelter called tatara, made by clay. When the smelter temperature is sufficiently high, the oxygen introduced by the bellows reacts with the carbon present in the charcoal to form carbon monoxide. The iron oxide in the ore (iron sand) then reacts with carbon monoxide to form pure iron and carbon dioxide, which escapes as gas. In tatara process the steel produced is an inhomogeneous mass of steel that can then be sorted out according to its carbon level and used for sword making [6]. The Japanese swords are produced from this tamahagane, featured by low carbon content which is wrapped by skin steel with high carbon. Then the system of the two steels is forged to produce a high number of layers by successive forge/fold operations. The forged steel is coated by a layer of clay mixed with charcoal. The clay coating is thinner near the blade, so that after heating up to $800^{\circ}-850^{\circ} \mathrm{C}$ and the following plunging in the water, the blade can undergo a quenching process in order to produce the martensite. The blade zone undergoes the phase transition which causes the martensitic phase in the fast cooled region and the related thermal expansion causes the particular bended shape of the typical Japanese swords. Swords of different size and shape are found in NBTHK (Nihon Bijutsu Token Hozon Kyokai-The society for preservation of Japanese Art Sword) which works on preservation, improvisation and study of the antique swords [7].

a e-mail: ohba@riko.shimane-u.ac.jp

This is an Open Access article distributed under the terms of the Creative Commons Attribution-Noncommercial License (http://creativecommons.org/licenses/by-nc/3.0/), which permits unrestricted use, distribution, and reproduction in any noncommercial medium, provided the original work is properly cited. 
Inasmuch as the mechanical properties of metallic materials are greatly affected by its microstructures. To study the fabrication of micro/nanostructured metallic materials is one of the important topics among the materials scientists. Martensite morphology forms through the displacive transformation in the crystal structure. The martensite in steel has mainly two types of morphologies, lenticular and lath martensite, depending on carbon content. The hierarchical substructure of lath marteniste including packets, blocks and laths is important. The packet size has also been reported to affect the toughness of martensitic steels $[8,9]$. Therefore, the effort to investigate the microstructure is necessary for better understanding of the cause of strength and toughness. In the present study we investigated the characteristic features of Japanese sword from microstructural point of view and discussed the results in the light of contemporary reports.

\section{Experimental Procedure}

The object of the present study is a short sword (knife) produced by Japanese sword maker (swordsmith) following the traditional recipe. The sword has been cut into pieces using a computer controlled spark wire cutter (Brother HSC-300) for different experimental observation.

The analysis of the microstructure has been performed on two cut sections: one for observing the cross-section and the other for surface area as shown in Fig.1 schematically. The preliminary observation of the microstructure has been done by optical microscopy after proper grinding and polishing of the extracted sections. Chemical etching has been performed by immersing the cut sections in the nital metallographic etching solution $\left(0.5 \% \mathrm{HNO}_{3}\right.$ for $100 \mathrm{~mL}$ of ethanol) for $3 \mathrm{sec}$. The chemical composition of the sword including nonmetallic inclusions has been measured by the SEM-

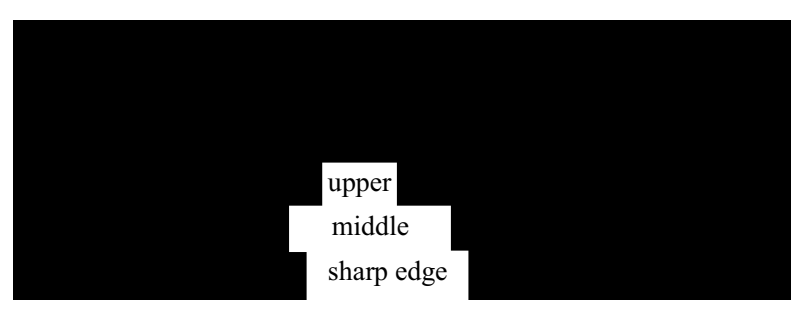
EDS technique.

The SEM-EBSD (Electron-Back-Scattering-Diffraction) experiment has been performed for observing the crystalline orientation of packet and blocks etc. The experiments were carried out on surface area and the cross-sectional area (middle) of the sword by using a JEOL-7001F Scanning Electron Microscope. For EBSD measurements and analyses OIM analysis-ver. 5.2 and TSL Solution Ltd. system have been used. The analyzed area is featured by a $60 \mathrm{~mm}^{2}$ extension and the area has been scanned using an accelerating voltage of $25 \mathrm{kV}, 15$ $\mathrm{mm}$ working distance and the step size chosen for each maps is of $0.02 \mu \mathrm{m}$ after tilting the sample $70^{\circ}$. A Vickers micro-=hardness test has been carried out to point out the trend of the micro-hardness values along the cross-section as a function of the depth from the lateral sword side.

\section{Results and Discussion}

\subsection{Optical Microscopy}

The observations have been performed on two different specimens to realize the microstructure of different parts of the sword. Fig. 2 show optical macroscopic image of the surface and a martensitic structure is seen in the indicated area of the adjacent figure.

Figs. 3(a), (b) and (c) show optical macroscopic images of three different zones of the cross-sectional sample. All these three images show a clear martensitic structure with a few dark non-metallic inclusions. The morphology of the martensite is appeared to be lath type. Yaso et. al, [10] have observed the cross-section of old Japanese sword made by second generation of Muramasa. They mentioned the structure near sharp edge is martensite whereas in other regions the structures are fine pearlite or coarse pearlite. In our observation we found that the overall structure of the sword is a lath martensite. It also may come from quenching speed difference; as our sword (short knife) is smaller than ordinary one.

The density of inclusions is also less than that mentioned by Yaso et al, [10]. These discrepancies may arise due to the different specimens prepared in different period of time. 


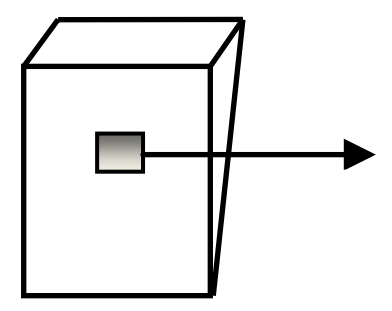

Fig. 2. Optical microscopic images of surface of Japanese sword. Arrow indicates horizontal direction in the right figure.

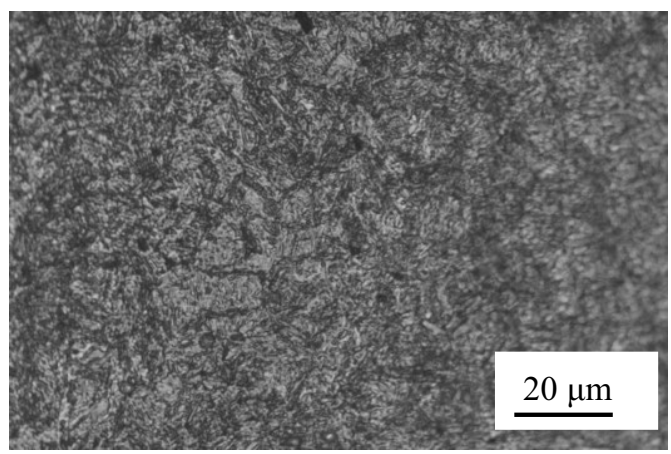

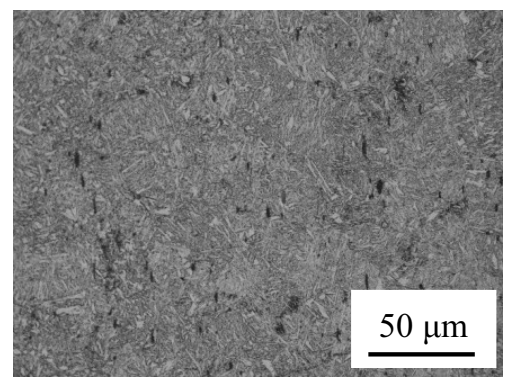

(a) upper zone

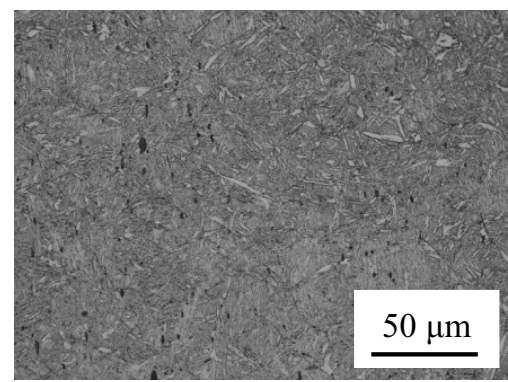

(b) Middle zone

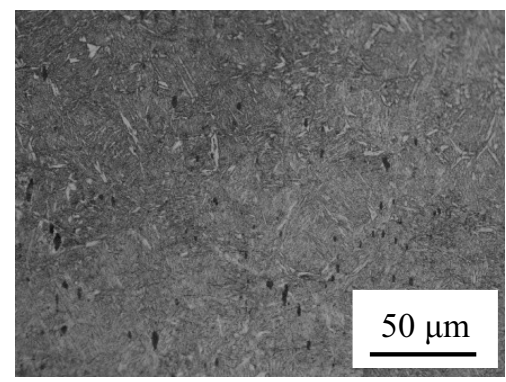

(c) Sharp edge

Fig. 3. Optical microscopic images of different zones of the cross-sectional specimen.

\subsection{SEM-EDS observation}

The chemical compositions of the inclusions are shown in Fig. 4 and Table 1. It appears from the EDS measurements that some non metallic oxide materials exist in the cross-section of the sword. The distribution of inclusions looks rather homogenous and the average size of each inclusion is limited within 5-10 $\mu$ m. Carbon concentration is found to be less than 0.5 mass \%, which is fewer than that in old sword studied by Yaso et al. [10]. Inclusions shown here are considered to come from the clay of Tatara smelter. They penetrate into Tamahagane and remained in the sword. Rather high existence of $\mathrm{TiO}_{2}$ is different from ordinary steel.

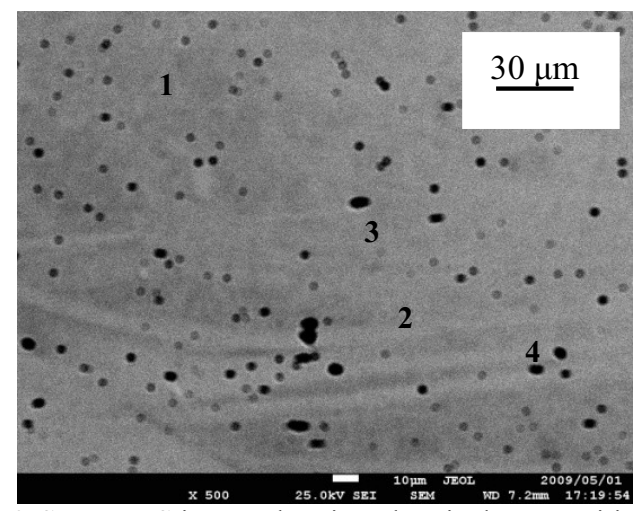

Fig. 4. SEM-EDS image showing chemical composition at different points in the cross-sectional part.
Table 1.

Point \# 1 and 2

\begin{tabular}{ll} 
Chemical forms & Mass\% \\
\hline $\mathrm{Fe}$ & 100
\end{tabular}

Point \# 3 and 4

Chemical forms $\quad$ Mass $\%$

$\mathrm{SiO}_{2} \quad 14.89$

$\mathrm{Al}_{2} \mathrm{O}_{3} \quad 4.01$

$\mathrm{TiO}_{2} \quad 2.72$

$\mathrm{K}_{2} \mathrm{O} \quad 1.94$

$\mathrm{CaO} \quad 0.80$

$\mathrm{Na}_{2} \mathrm{O} \quad 0.75$

$\mathrm{Fe} \quad 74.89$

The SEM-EBSD observations have been done on cross section and surface parts to elucidate the crystal orientation and to measure the packet and block size in the prior austenite grain. Fig. 5 shows the EBSD crystal orientation map of the cross-section near middle portion. The non-metallic inclusions (black points) are observed in the Fig. 5(a). Since we are interested in the microstructure including packet and block, the enclosed area of Fig. 5(a) has been analysed and are shown in Figs. 5(b). Fig. 5(b) shows the blocks within a packet; red, green and blue colours have been inserted to visualize the blocks within a packet of prior austenite grain. The average thickness of blocks has been estimated by the intercept method. It has been found that the average block thickness is $0.78 \mu \mathrm{m}$ and the packet size is about $15 \mu \mathrm{m}$.

It is realized that the density of non-metallic inclusions in surface are comparatively less than that of the cross-sectional area as shown in fig. 6(a). The average size of the inclusions is comparatively larger than that in 
cross-section. Fig. 6(b) shows the blocks within a packet. Average block thickness is determined $0.64 \mu \mathrm{m}$ and the packet size is $7 \mu \mathrm{m}$ in the surface area. The misorientation angle crossing one of block boundaries has been determined as $59.7^{\circ}$ (not shown figuratively).

We need further precise studies for detailed understanding of Japanese sword, we can say that the microstructure in Japanese sword is lath martensite. This is different from Damascus steel. Further, it is perceptible from this study that Japanese swordsmiths utilized lath martensite without knowing the details about lath in old days which are explained by the researchers these days.

\subsection{Vickers hardness}

Vickers micro-hardness has been measured on the cross-sectional part to realize the hardness in different part of the sword. It has been found that the hardness of the sharp edge is very high $(866 \mathrm{Hv})$ and the middle portion is little less hard $(787 \mathrm{Hv})$ than other portions. It might be due to the less concentration of carbon in
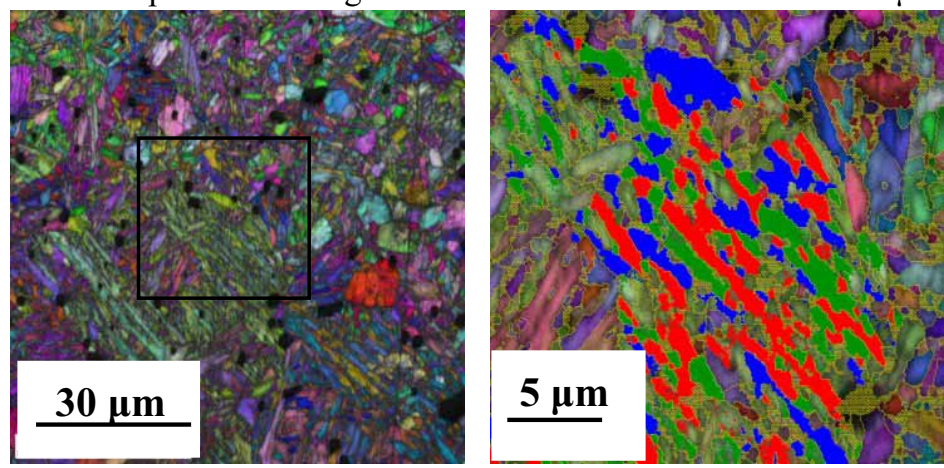

Fig. 5. (a) Crystal orientation map of the central part in the cross section: (b) shows the blocks and in a packet in the selected area of image.
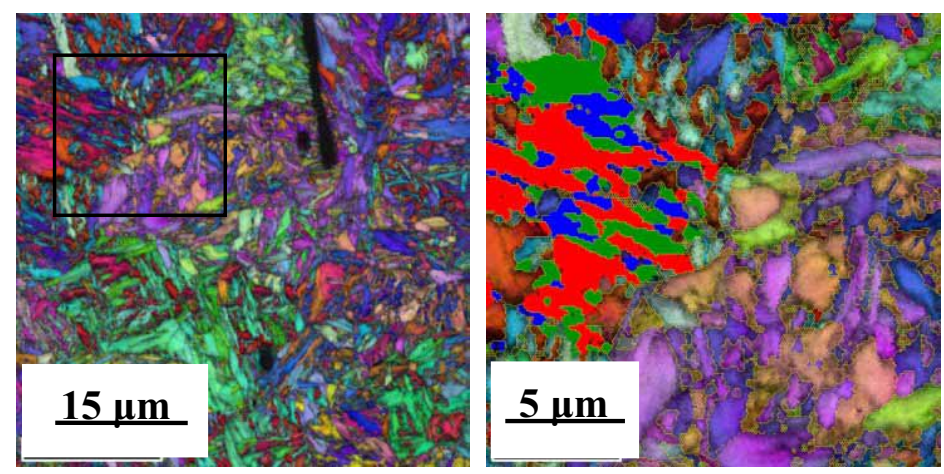

Fig. 6. (a) Crystal orientation map of the surface: (b) shows the blocks in a packet in the enclosed area of image (a). the middle portion. The microscopic observation by SEM indicated that the packet size of the middle zone of the cross section is larger than that of surface region. The fact support that carbon concentration in the surface is higher than that in the cross section [11].

\section{Summary}

Micro structure of a newly prepared Japanese sword was characterized crystallographically using OM, EDS and EBSD with FE-SEM. The lath martensite structure was found in either on the surface and cross-section of the sword. Packet sizes revealed that carbon concentration in cross section is less than that on the surface. As the carbon content increases the size of packet decreases [11]. The density distribution of inclusions (oxides) in the cross- sectional area is higher than that in the surface area. It might be due to the traditional making procedure (tatara) of the sword. Vickers micro hardness is found very high in the sharp edge and comparatively lower in the middle portion of the cross section. Present study elucidates that the tatara iron and the manufacturing procedure gave distinctive features to Japanese swords which is different from Damascus steel and the Japanese swordsmith utilized lath martensite without knowing details about it.

\section{Acknowledgement}

The present work was financially supported by Tatara project of the Ministry of Education, Science and Culture of Japan Government and the Organization for the Promotion of Project Research in Shimane University. We also acknowledge Mr. Sadanao Mikami, who is one of famous Japanese sword blacksmiths, for producing the short knife (sword) for the present research. 


\section{References}

[1] M. Reibold, P. Paufler, A. A. Levin, W. Kochmann , N. Patzke and D. C. Mayer, NATURE, Vol. 444, 286(2006)

[2] Carlo MAPELLI, Walter NICODEMI and Riccardo F. RIVA, ISIJ International, 47, 1050 (2007)

[3] R. F. Tylecote and B. J. J. Gilmour: The Metallurgy of Early Ferrous Edge Tools and Edged Weapons, BAR Brit. Ser. 155, Oxford, (1986) p.77

[4] T. Inoue, Mater. Sci. Res. Int., 3, 193 (1997)

[5] Wadsworth, J. MRS Bull. 27, 980(2002)

[6] Leon and Hiroko Kapp and Yoshindo Oshihara, The Craft of the Japanese Sword - by Kodansha International Ltd. Tokyo Japan, First edition, (1987) p. 30.

[7] Krauss G. Steels heat treatment and processing principles. Materials Park (OH): ASM International; 1990.

[8] Oleg D. Sherby, Jeffrey Wadsworth, Donald R. Lesuer and Chol K.Syn, Materials Transactions, 49, 2016 (2008).

[9] Hiromoto Kitahara, Rintaro Ueji, Nobuhiro Tsuji and Yoritoshi Minamino, Acta Materialia, 54 1279(2006)

[10] Muneo YASO, Toshifumi TAKAIWA, Yoshihiro MINAGI, Kunichika KUBOTA, Shigekazu MORITO, Takuya OHBA and A. K. Das, 'Study of microstructure on cross section of Japanese sword', submitted in ESOMAT-2009, Prague, Czech Republic.

[11] S. Morito, H. Tanaka, R. Konishi, T. Furuhara and T. Maki, Acta Materiala , 51, 1789 (2003). 